\title{
Energy dissipation due to breakage during confined compaction of granular materials
}

\author{
Brett Kuwik ${ }^{1, *}$ and Ryan C. Hurley ${ }^{1, * *}$ \\ ${ }^{1}$ Department of Mechanical Engineering, Johns Hopkins University, Baltimore, MD 21218, USA
}

\begin{abstract}
The dissipation of energy during the compaction of granular materials was studied by performing confined drop tower experiments on Ottawa sand. Energy dissipated due to breakage was quantified by evaluating the creation of new surfaces at varying drop heights. Post-compaction particle size distributions (PSD) were measured and the amount of breakage was quantified by the position of the current PSD relative to the pre-compaction and ultimate PSD. Our observations revealed that the percentage of input energy dissipated due to breakage accounted for less than $0.5 \%$ of the total energy budget and was a constant proportion regardless of the total energy applied to the system. We also evaluated the effects of die wall friction by measuring post-compaction PSD in various positions within the sample.
\end{abstract}

\section{Introduction}

Granular materials are ubiquitous in nature and are studied across multiple disciplines including food production, ceramic processing, and pharmaceutical manufacturing. Research on the compaction of granular materials has noted that multiple mechanisms dominate the mechanical response. During the initial stages of compaction, granules rearrange to form a more ordered packing structure. Friction between particles dominates in this stage of loading. As loading continues, the yield point is reached and particles begin to fracture, causing a decrease in the bulk porosity and allowing newly created particles to rearrange. This stage of loading is dominated by fracture and friction between particles. The different regimes of powder compaction have been well documented and previous literature has observed dominant mechanisms of fracture and friction [1]. However, issues that remain unresolved include quantifying the amount of particle breakage and understanding how much energy is being dissipated to fracture, friction, thermal effects, and other mechanisms.

The total energy balance of an impact event can be written as [2]:

$$
\delta W=\delta \Psi_{e}+\delta \Phi_{p}+\delta \Phi_{S}+\delta \Phi_{\text {redist }}
$$

where $\delta \Psi_{e}, \delta \Phi_{p}, \delta \Phi_{S}$, and $\delta \Phi_{\text {redist }}$ represent the elastic energy, plastic dissipation energy, breakage energy and redistribution energy, respectively. This equation describes how all energy supplied to a system is stored elastically or dissipated through various mechanisms. For granular materials, elastic stored energy is limited and the other mechanisms consume most of the supplied energy. Pre-

\footnotetext{
*e-mail: bkuwik1@jhu.edu

**e-mail: rhurley6@jhu.edu

A video is available at https://doi.org/10.48448/pq70-2z06
}

vious researchers have examined breakage of granules [35], but limited work has quantified the amount of energy dissipated due to breakage during the different stages of compaction, particularly during rapid impact.

Many theories have been proposed to quantify the breakage of granular materials using post mortem analysis of particle sizes. One classical theory uses data of particle size distribution (PSD), a common laboratory measurement [6]. In this theory a relative breakage index variable $\mathrm{Br}$ is defined and quantifies the amount of breakage relative to the amount of possible breakage:

$$
B r=\frac{B_{\text {total }}}{B_{\text {Potential }}}, \quad 0 \leq B r \leq 1,
$$

where zero indicates no breakage has occurred and one is the the maximum allowable breakage. Total breakage is calculated as the area between the current and initial PSD and breakage potential is the area between initial and ultimate PSD:

$$
B_{r}=\frac{\int_{d_{m}}^{d_{M}}\left(\left(F(d)-F_{0}(d)\right) d^{-1} d d\right.}{\int_{d_{M}}^{d_{m}}\left(\left(F_{u}(d)-F_{0}(d)\right) d^{-1} d d\right.},
$$

where $d_{m}$ is the smallest grain size, $d_{M}$ is the largest grain size, $F_{0}(d)$ is the initial PSD, $F(d)$ is the current PSD, and $F_{u}(d)$ is the ultimate PSD. The ultimate PSD is defined as:

$$
F_{u}(d)={\frac{d}{d_{M}}}^{3-\alpha},
$$

where $\alpha$ is the fractal dimension assumed to be 2.6 [7]. The exact ultimate PSD cannot be known unless reached experimentally. Nevertheless, the assumed ultimate PSD and corresponding fractal dimension agrees with observations [6]. 
The goal of this paper is to examine the dissipation of energy due to fracture as a function of relative breakage during the dynamic compaction of granular materials. Drop tower experiments were performed on Ottawa sand and PSD measurements were used to define relative breakage. The dissipation of energy was compared with the breakage index and total energy budget. In addition the breakage of particles at different heights from within the die were examined to study the effect of die wall friction.

\section{Experimental Techniques}

\subsection{Material}

Ottawa sand graded according to ASTM C778 (U.S. Silica Company, Ottawa, Illinois) was used in these experiments. Before being mechanically tested, the Ottawa sand was sieved to remove fines. Particles that were retained between No. $35(500 \mu \mathrm{m})$ and No. $70(212 \mu \mathrm{m})$ were used in the experiments. The size distribution of particles is summarized in Table 1.

Table 1. Particle size distribution characteristics post sieving

\begin{tabular}{ccccc}
\hline $\mathrm{D}_{10}[\mu \mathrm{m}]$ & $\mathrm{D}_{50}[\mu \mathrm{m}]$ & $\mathrm{D}_{90}[\mu \mathrm{m}]$ & Cir. & AR \\
\hline 363 & 463 & 577 & 0.94 & 0.77 \\
\hline
\end{tabular}

\subsection{Particle Measurement}

Particle sizes were measured using 2D images obtained using Malvern Morphologi 4 ID. In this instrument, particles were suspended in air and dispersed on a glass slide. A series of objective lenses scanned the dispersed particles to obtain 2D projection images of particles and a built-in program analysed the images to measure the major, minor, circle equivalent (CE) diameter, and other metrics to quantify particle morphology. These measurements were verified using 3D X-ray computed tomography (XRCT) as discussed below.

For 2D image projections, only two measurements of particle diameter are made: major and minor diameter. No measurement is made perpendicular to the projection plane and an assumption has to be made to calculate the volume and the surface area of particles. For volume measurements, 2D area projections were used and an assumed out plane diameter of the CE diameter was used. The volume of each particle was calculated by:

$$
\text { Vol }_{\text {particle }}=\frac{2}{3} * C E \text { diameter } * \text { Projected } 2 D \text { Area } \text {. }
$$

A similar method was used for calculating the surface area of particles. The perimeter of $2 \mathrm{D}$ projections was used and an assumed out of plane particle diameter of the $\mathrm{CE}$ diameter was used. The equation used to calculate the surface area of particles was:

$$
S A=2 * \text { Perimeter } * C E \text { Diameter. }
$$

Particles were also examined using an RX Solutions EasyTom 150/160 MicroCT. 3D images were segmented to separate individual particles. The surface area and the volume of the particles was calculated using built-in MATLAB (C) function "regionprops3".

To construct PSD curves, two different MATLAB C shape measurement methods were used to examine the shape of particles: "Principal Axis Length" and "Eigenvalues". For "Principal Axis Length", an ellipsoid that has the same normalized second central moments as the particle is fit to each particle and the principal axis length and directions are returned. "Eigenvalues" method finds the eigenvalues and eigenvectors that represent the voxels of the segmented particle. Each method returns three unique eigenvectors and eigenvalues for each particle. The eigenvectors determine the orientation of the particles principal axis and the eigenvalues determine the size of the corresponding axis.

\subsection{Configuration}

In the confined uni-axial compression experiments, a die sleeve confined the lateral expansion of the Ottawa sand as platens inserted at the top and bottom of the die compressed the sample. The top and bottom platen were fabricated from C350 Vascomax maraging steel and the die sleeve with an inner diameter and out diameter of 0.21 " and 0.50 " was fabricated from 304 stainless steel. Samples were placed in an Instron CAEST 9350 drop tower. The mass and height of the impact carriage were controlled to change the energy supplied to compress the sample.

During specimen preparation, one end of the die was closed and Ottawa sand was poured in from the top. To limit the effects of wall friction, samples were poured to a height equal to the inner diameter of the die for an aspect ratio of 1:1. Mass and height of samples were measured pre- and post-compaction for initial density and residual strain measurements.

Hydrostatic pressure was calculated by measuring the axial stress recorded from the drop tower impact rod and radial stress from a strain gauge mounted on the outside sleeve of die. To determine the radial stress in the sample, the die wall was assumed to be thick-walled and in a state of plane strain. Given material properties and die geometry, the axial stress along the inside of the die can be calculated and has previously been reported in the literature as [8]:

$$
\sigma_{\text {hyd }}=\frac{1}{3}\left(\sigma_{z}+\frac{\left(b^{2}-a^{2}\right) \epsilon_{d i e} E_{d i e}}{\left(1-v_{d i e}\right) a^{2}}\right),
$$

where $\sigma_{z}$ is the applied stress from drop tower, $b$ is the outer radius of the die, $a$ is the inner radius of the die, $v_{\text {die }}$ is the Poisson's ratio of the die, and $E_{\text {die }}$ is the Young's modulus of the die. 


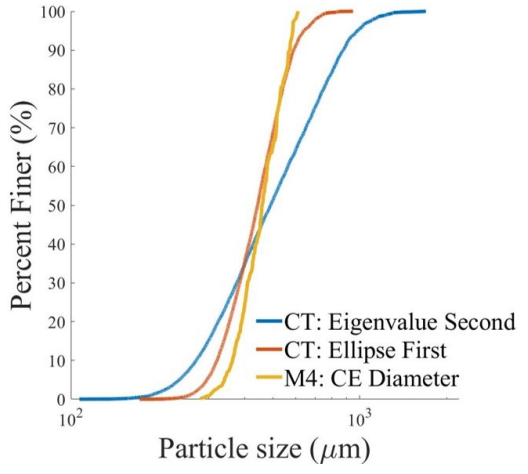

Figure 1. Morphologi 4 ID compared to XRCT. "Eigenvalue Second" assumes particle diameter of second largest axis length using "Eigenvalues" method. "Ellipse first" assumes particle diameter of largest axis using "Principal Axis Length"

\section{Experimental Outcomes}

\subsection{D image projections vs 3D particles}

We first compared the measurement of PSD using 2D image projections from Morphologi with 3D XRCT measurements. Previous research has noted a difference between 2D projections and 3D images of particles and has proposed methods for corrections between the two methods [9-10]. As particles are dispersed for 2D measurements, particles are assumed to all rest on the same axes. However, the axes that particles rest on is unknown. Thus, PSD from 3D XRCT were measured using two methods described in Section 2.3. In Figure 1, the eigenvalues from each method are compared with Morpholgi 4 ID PSD. It can be seen the largest eigenvalue from "Principal Axis Length" fits the morphology data the best and illustrates how all particles are resting on the same axis.

Next, we evaluated the accuracy of using CE diameter for the out of plane diameter in surface area and volume measurements. As seen by Table 2, the specific surface area (SSA) measured by Morphologi 4 ID and XRCT are close to one another, confirming the accuracy of the SSA computed from the Morphologi data. A similar trend is observed for circularity/sphericity and aspect ratio measurements.

Table 2. Comparison between 2D particle projections and 3D particle shape

\begin{tabular}{ccc}
\hline & Morphologi 4 ID & XRCT \\
\hline SSA $\left(\frac{m^{2}}{g}\right)$ & 0.01007 & 0.00723 \\
\hline Circularity/Sphericity & $0.94 \pm 0.02$ & $0.85 \pm 0.03$ \\
\hline Aspect Ratio & $0.77 \pm 0.06$ & $0.59 \pm 0.06$
\end{tabular}

\subsection{PSD}

We next investigated the evolution of the PSD as a function of drop height. As shown by Figure 2a, pre- and post- compaction PSD were measured using Morphologi
4 ID. In the drop tower, the mass of the falling carriage was kept constant and the drop height was varied between 50 and $700 \mathrm{~mm}$. It can be seen that as the drop height increases, post-compacted PSD curves broaden, signifying the creation of smaller particles. The monotonic broadening of PSD illustrates that for all performed drop tower experiments the ultimate particle size distribution is never reached. It should also be noted that each PSD can be fitted to a power-law and similar monotonic increasing of the power law exponent is observed.

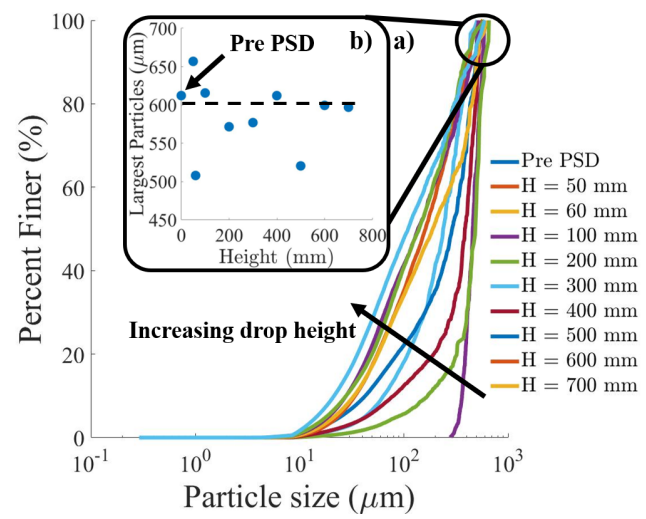

Figure 2. (a) PSD at varying heights, (b) largest particle from each sample plotted vs drop height

As illustrated in Figure 2b, we examined the largest particle the Morphologi 4 ID measured from each sample. There is no relation between largest particle and drop height, suggesting that the largest particle does not break during our experiments. This finding is counter intuitive because larger particles have larger and more internal defects than smaller particles, making them easier to fracture. However, during initial stages of compaction the large granules will start to fracture, creating smaller granules. Smaller particles fill in voids around larger particles, increasing the packing efficiency. The smaller particles also act to transform a small number of high intensity contact forces from pre-compaction to many lower intensity contact forces, thus creating a "cushion" effect [11]. The cushion causes larger particles to be in hydrostatic state of compression, causing internal defects to be in compression. Under compression loading, micro cracks will not necessarily propagate, mitigating the effects of larger microcracks in larger particles.

\subsection{Dissipation of Energy}

Next, we investigated the breakage index to quantify the amount of breakage as a function of drop height. As seen in Figure 3a, total breakage was calculated using Eq. 3 and the pre- and post-compaction PSD and an assumed ultimate PSD. As can be observed, the amount of breakage, $\mathrm{Br}$, is not linearly related to drop height, but instead is observed to plateau as drop height increases.

In addition, we investigated the portion of the energy budget consumed by breakage as a function of input energy. Energy dissipated by fracture was calculated by multiplying the amount of newly created surface area within 

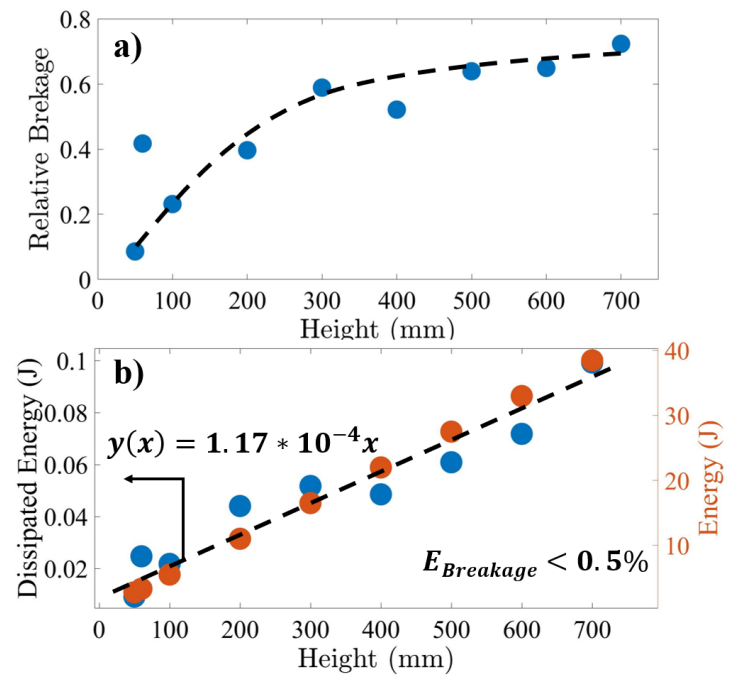

Figure 3. (a) Relative breakage plotted against drop height, (b) energy dissipated by creation of surfaces and total energy from drop tower plotted vs drop height

the sample and the known surface energy density of Ottawa sand $\left(15.0 \mathrm{~J} / \mathrm{m}^{2}\right)$. Total input energy was calculated by using the mass and the drop height of the carriage impacting the sample. As seen in Figure 3b, the percentage of dissipation in the creation of surfaces is less than $0.5 \%$ of the complete energy budget. A small dissipation of energy due to breakage implies that other dissipation mechanisms such as friction and thermal effects are more dominant factors in the dissipation of energy. Another interesting aspect of Figure $3 b$ is the linear relationship between dissipation of energy by creation of surfaces and the drop height. A constant portion of energy is consumed by fracture, even as a nonlinear breakage index is illustrated in Figure 3a.

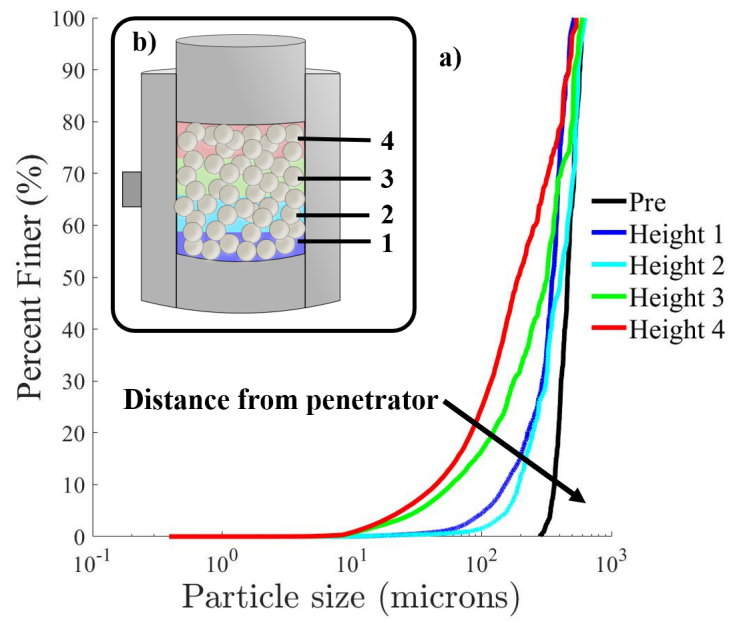

Figure 4. (a) PSD post-impact at different heights inside die, (b) illustration of varying heights within die

\subsection{Die wall friction}

Next, we investigated the effects of die wall friction by examining the PSD at various heights within the die post- compaction. As illustrated by Figure 4, four different regions were sampled within the die, with height 4 being closest to the region of impact and height 1 being farthest away from the impact region. Granules from within each section were measured using the Morphologi 4 ID. It can be observed that granules measured closer to the top platen (closer to impact region) had a broader PSD with more fines than samples near the bottom of the sample. With the PSD curves, the breakage index was calculated with an assumed ultimate size distribution. A similar relationship was observed: breakage index decreased as sampled further away form the top platen. The difference of breakage as a function of height is also observed in quasi-static compaction and is caused by die wall friction [12]. For dynamic compaction experiments, attenuation of the shock wave can also cause a change in breakage as a function of height.

\section{Conclusion}

We have shown that, during dynamic compaction of granular materials, PSD can be used to determine the amount of breakage using a relative breakage index variable. We have also shown that a fixed portion of the total energy budget is dissipated by fracture. This portion accounts for less than $0.5 \%$ of the entire energy budget. Thus, other mechanisms dissipate the majority of the energy. We further showed the effects of die wall friction and changes in PSD as a function of height with in the sample.

Part of this work was sponsored by the Department of the Defense, Defense Threat Reduction Agency under award HDTRA1-20-2-0001. The content of the information does not necessarily reflect the position or the policy of the federal government, and no official endorsement should be inferred.

\section{References}

[1] R.W. Heckel, T Metall Soc AIME 221, 671-675 (1961)

[2] A.R. Russell, Geotech Lett 1, 5-9 (2011)

[3] J. Huang, S. XU, S. Hu, Int J Impact Eng 59, 1-10 (2013)

[4] D. Gong, S. Nadolski, C. Sun, B. Klein, J. Kou, Powder Technol 339, 595-605 (2018)

[5] Y. Xiao, Z. Yuan, Y. Lv, L. Wang, H. Liu, Constr Build Mater 2018, 188-199 (2018)

[6] I. Einav, J Mech Phys Solids 55, 1274-1297 (2007)

[7] M.R. Coop, I.K. Lee, Proceedings of Wroth Memorial Symposium, 186-198 (1993)

[8] M. H. Sadd, Elasticity (Academic Press, 2009)

[9] T. Ueda, T. Oki, S. Koyanaka, Powder Technol 343, 287-295 (2019)

[10] D. Sun, W.M. Yan, Acta Geotechnica 15, 1533-1555 (2020)

[11] O. Ben-Nun, I. Einav, Philos T R Soc A 368, 231247 (2010)

[12] B.J. Briscoe, S.L. Rough, Colloid and Surface 137, 103-116 (1998) 